\title{
Análise da camada de fibras nervosas pelo GDx em pacientes pseudofácicos com opacidades da cápsula posterior
}

\author{
Analysis of the nerve fiber layer using GDX in pseudophakic patients with \\ posterior capsular opacification
}

Tatiana Azevedo Arraes ${ }^{1}$

Hellmann Dantas Cavalcanti ${ }^{2}$

João Arraes $^{3}$

Ana Cecília de Souza Leão ${ }^{4}$

Marilia Fernandes Sena ${ }^{5}$

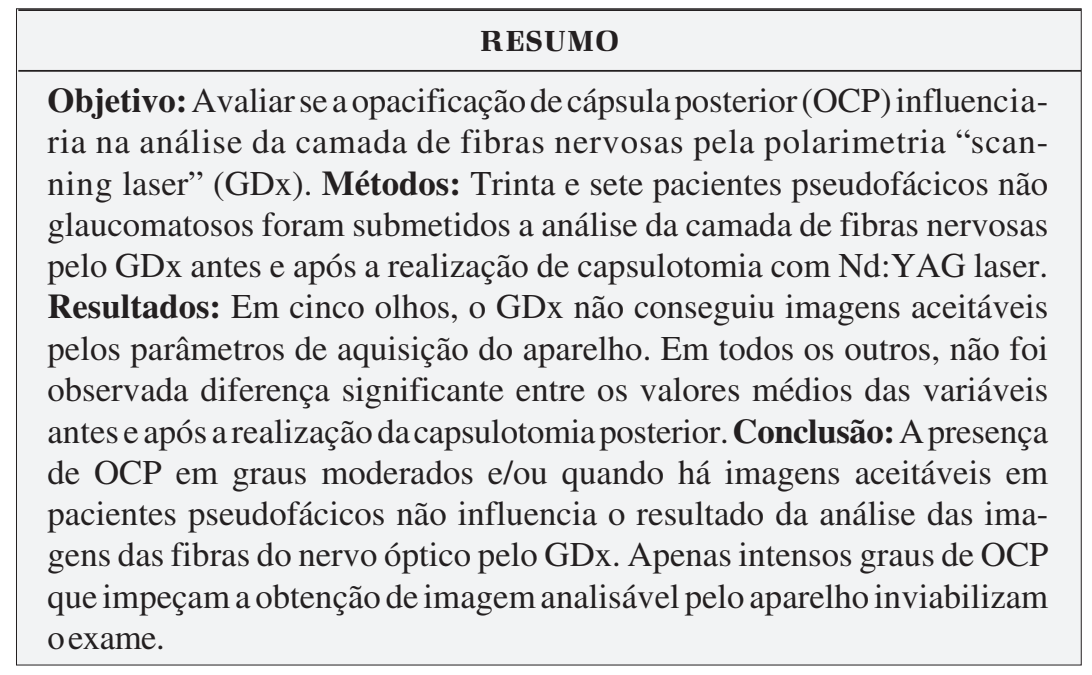

Descritores: Pseudofacia; Glaucoma; Fibras nervosas/patologia; Técnicas de diagnóstico oftalmológico; Lasers/uso diagnóstico

fe (PE) - Brasil.

Especialista em Córnea e Doenças Externas Oculares pelo Centro de Referência em Oftalmologia (CEROF) do Hospital das Clínicas da Universidade Federal de Goiás - UFG - Goiânia (GO) - Brasil.

Oftalmologista do Departamento de Glaucoma da Fundação Altino Ventura - Recife (PE) - Brasil.

${ }^{3}$ Pós-graduando do Centro de Ciências da Saúde da Universidade Federal de Goiás; Especialista em Retina e Vítreo pelo CEROF do Hospital das Clínicas da UFG Goiânia (GO) - Brasil.

Especialista em Oftalmologia pelo Conselho Brasileiro de Oftalmologia - Fundação Altino Ventura - Recife (PE) - Brasil.

${ }^{5}$ Especialista em Oftalmologia pelo Conselho Brasileiro de Oftalmologia - Fundação Altino Ventura - Recife (PE) - Brasil.

Endereço para correspondência: João Carlos Diniz Arraes. Quadra 110 Sul - Alameda 7 - Lote 1ํㅡ - Casa 9 Palmas (TO) CEP 77020-132

E-mail: joaoarraes@hotmail.com

Recebido para publicação em 01.05.2006

Última versão recebida em 01.10 .2007

Aprovação em 10.10.2007

Nota Editorial: Depois de concluída a análise do artigo sob sigilo editorial e com a anuência da Dra. Maria Rosa Bet de Moraes e Silva sobre a divulgação de seu nome como revisora, agradecemos sua participação neste processo.

\section{INTRODUÇÃO}

Com o progressivo aumento da expectativa de vida, a ocorrência simultânea de doenças relacionadas à senilidade, como glaucoma e catarata, é cada vez mais freqüente ${ }^{(1)}$. A cirurgia de catarata, portanto, torna-se muitas vezes necessária em pacientes portadores de glaucoma ${ }^{(2)}$ e a principal complicação desta cirurgia é a opacificação da cápsula posterior ${ }^{(3-8)}$.

Um defeito na camada de fibras nervosas $(\mathrm{CFN})$ da retina representa um achado precoce do glaucoma, precedendo as alterações biomicroscópicas no nervo óptico ${ }^{(9)}$ e campo visual ${ }^{(10)}$. O laser confocal polarizado (GDx) quantifica, in vivo, a camada peripapilar de fibras nervosas, através da análise da birrefringência que os axônios das células ganglionares apresentam à luz polarizada. Devido a sua grande reprodutibilidade ${ }^{(11)}$ e sensibilidade $^{(12-15)}$, o laser confocal polarizado pode ser útil no estudo populacional do glaucoma ${ }^{(16)}$.

A aquisição das imagens pelo GDx em doenças que diminuem a transparência dos meios intra-oculares pode resultar em pobre associação com a clínica, pobre reprodutibilidade e imagens irreais ${ }^{(17)}$. No entanto, após minuciosa busca na literatura científica dos últimos dez anos, não foram encontradas informações sobre o possível efeito da opacificação da cápsula posterior em pseudofácicos na análise da CFN pelo GDx.
} 
Com o intuito de contribuir na decisão de indicações deste exame na propedêutica do glaucoma em pacientes operados de catarata, foi objetivo deste estudo avaliar possíveis diferenças na análise da CFN pelo GDx em pacientes pseudofácicos induzida por diferentes graus de opacificação de cápsula posterior $(\mathrm{OCP})$.

\section{MÉTODOS}

Foi realizado um estudo com 45 olhos (22 olhos direitos e 23 olhos esquerdos) de 37 pacientes pseudofácicos do Ambulatório de Catarata da Fundação Altino Ventura no período entre maio e dezembro de 2003.

Foram incluídos pacientes pseudofácicos com opacificação de cápsula posterior, não glaucomatosos, com idade variando entre 30 e 89 anos (média=69 anos; $\mathrm{DP}=15,2$ anos), sendo $60 \%$ do gênero feminino. A cirurgia da catarata foi realizada pelas técnicas extracapsular ou facoemulsificação com implante de lente intra-ocular. A quantificação da OCP foi feita na lâmpada de fenda e seguiu a seguinte classificação: Grau 1, OCP presente, mas com nítida visualização do fundo do olho e de detalhes do nervo óptico (NO) e mácula (M); Grau 2, OCP presente, visualização de fundo de olho dificultada, mas ainda possível visualizar alguns detalhes do $\mathrm{NO}$ e $\mathrm{M}$; Grau 3, OCP presente, visualização de fundo de olho dificultada, NO e M visíveis, mas com dificuldade e sem definir detalhes; Grau 4, OCP presente, com visualização apenas de sombra de NO e retina; Grau 5, OCP presente e impossível visualização de fundo de olho. Todos os pacientes foram submetidos a perimetria computadorizada (técnica 24-2) um mês após a realização da capsulotomia.

Foi definido como não glaucomatoso aquele paciente que apresentava pressão intra-ocular menor que $21 \mathrm{mmHg}$; nervo óptico com escavação papilar menor ou igual a $40 \%$ da área total do disco, assimetria menor que $20 \%$ em relação ao olho adelfo e sem outros sinais glaucomatosos; e campo visual computadorizado (técnica 24-2), realizado um mês após a capsulotomia, sem sinais sugestivos de glaucoma.

Foram excluídos os pacientes com alterações na transparência dos meios ópticos que não fossem exclusivamente decorrentes da OCP, outras doenças do nervo óptico e da retina, ou ainda quando a perimetria no $1^{\circ}$ mês pós-operatório mostrava alguma alteração sugestiva de glaucoma ou outra neuropatia. Quando um dos olhos de um mesmo paciente tinha sua escavação papilar considerada como suspeita de glaucoma, automaticamente o olho adelfo também foi excluído do estudo.

Todos os pacientes foram avaliados pelo mesmo examinador. Dos 45 olhos estudados (37 pacientes), em 5 olhos ( 3 pacientes), o GDx não conseguiu obter imagens devido ao intenso grau de OCP (4 olhos com Grau 4 e 1 olho com grau 5). Dos 40 olhos restantes, a frequiência de OCP foi a seguinte: 6 olhos com OCP G1, 24 olhos com OCP G2 e 10 olhos com OCP G3.

Os pacientes realizaram exame de GDx (NFAII/GDx - Laser
Diagnostic Technologies, San Diego, USA) e logo a seguir foram submetidos à capsulotomia com o Nd:YAG laser. A capsulotomia foi realizada apenas no eixo visual ( 3 a $4 \mathrm{~mm}$ ) em forma de "árvore de natal" e após sua realização foram prescritas medicações para o controle da pressão intra-ocular e inflamação intra-ocular por 7 dias. Não houve complicações significativas após o procedimento. O exame da análise da camada de fibras foi repetido após 15 dias utilizando o mesmo GDx. Foram obtidas 2 imagens de cada olho em cada exame, aceitas pelos critérios de aquisição do aparelho, e somente considerada para estudo a imagem média destas. Todos os parâmetros do aparelho foram incluídos para comparação e análise dos resultados. Foram consideradas como satisfatórias as imagens aceitas pelos critérios de aquisição do aparelho.

Foram analisados os parâmetros médios e respectivos desvios-padrão obtidos na análise da CFN pelo GDx antes e após a realização da capsulotomia com Nd:YAG laser dos seguintes valores: Q, Superior, Nasal, Inferior, Temporal, Número de variáveis alteradas, Assimetria, "Superior Ratio", "Inferior Ratio", Superior/Nasal, "Max Modulation", "Ellipse Modulation", Número, "Average Thickness", "Ellipse Average", "Superior Average", "Inferior Average" e "Superior Integral". Foi também analizado o valor mediano e suas respectivas distâncias interquartílicas da acuidade visual antes e após a realização da capsulotomia.

Foi utilizado o teste t-Student para amostras pareadas na análise de possíveis diferenças entre médias dos parâmetros do GDx e o teste de Wilcoxon para possível diferença entre os valores medianos da acuidade visual antes e após a realização da capsulotomia. $\mathrm{O}$ valor de $\mathrm{p}<0,05$ rejeitou a hipótese de nulidade.

\section{RESULTADOS}

Nos 40 olhos em que a análise da CFN pelo GDx foi possível, a comparação das imagens obtidas não mostrou em nenhuma das variáveis diferença significante entre os valores médios antes e após a capsulotomia com Nd:YAG laser. Apenas a acuidade visual teve diferença significante entre seus valores medianos antes e após a capsulotomia posterior. (Tabela 1).

\section{DISCUSSÃO}

A obtenção da imagem da camada de fibras nervosas (CFN) da retina é conseguida pelo analisador de fibras nervosas (GDx) a partir da birrefringência que os axônios das células ganglionares apresentam à luz polarizada. Esta ao passar através da CFN da retina sofre um retardo proporcional à espessura $^{(18)}$.

O analisador de fibras nervosas GDx possui um elemento óptico compensador para anular a birrefringência da córnea 
Tabela 1. Distribuição dos parâmetros do GDx e respectivos valores médios com desvios-padrão antes e após a capsulotomia posterior com Nd:YAG laser

\begin{tabular}{|c|c|c|c|c|}
\hline \multirow[t]{2}{*}{ Variáveis } & \multicolumn{3}{|c|}{ Valor médio / mediano } & \multirow[t]{2}{*}{$\mathbf{P}$} \\
\hline & \multicolumn{2}{|c|}{ Antes da CP } & Após a CP & \\
\hline Q & 82,40 & $\pm \quad 4,40 \%$ & $85,20 \pm 5,40 \%$ & 0,15 \\
\hline Nasal & 9,50 & $\pm 16,70$ & $7,30 \pm 14,40$ & 0,17 \\
\hline Inferior & $-4,90$ & $\pm 21,70$ & $-4,30 \pm 17,80$ & 0,72 \\
\hline Temporal & 0,62 & $\pm 17,40$ & $-0,31 \pm 14,80$ & 0,52 \\
\hline "Superior ratio" & 1,80 & $\pm \quad 0,38$ & $1,80 \pm 0,32$ & 0,73 \\
\hline "Inferior ratio" & 1,93 & $\pm \quad 0,34$ & $1,97 \pm 0,30$ & 0,40 \\
\hline Superior/nasal & 1,66 & $\pm \quad 0,26$ & $1,70 \pm 0,24$ & 0,38 \\
\hline "Max modulation" & 1,04 & $\pm \quad 0,38$ & $1,04 \pm 0,33$ & 0,95 \\
\hline "Ellipse modulation" & 1,74 & $\pm \quad 0,51$ & $1,89 \pm 0,50$ & 0,13 \\
\hline "Inferior average" & 75,80 & $\pm 20,10$ & $76,00 \pm 16,20$ & 0,87 \\
\hline "Superior integral" & 0,18 & $\pm \quad 0,04$ & $0,17 \pm 0,03$ & 0,66 \\
\hline
\end{tabular}

humana. Já foi provado, também, que o cristalino em seu estado translúcido, tem efeitos negligenciáveis sobre o resultado deste exame ${ }^{(19-20)}$.

A influência da catarata na polarimetria "scanning laser" pode levar a uma redução na qualidade da imagem, subestimando a camada de fibras nervosas em cataratas densas, particularmente na subcapsular posterior ${ }^{(20-21)}$. Sabe-se que a extração da catarata com implante de LIO não tem efeito significante nas medidas da polarimetria com GDx ${ }^{1}$.

É de grande importância, também, avaliar se alterações na transparência e/ou refratométricas dos meios ópticos poderiam falsear os resultados da análise da CFN pelo GDx. O conhecimento das limitações desta tecnologia permite o seu uso de forma mais segura e apropriada na prática clínica ${ }^{(17)}$.

Algumas doenças do segmento anterior e posterior podem, de fato, dificultar a aquisição da imagem como edema, transplante de córnea, precipitados ceráticos, uveíte anterior, cataratas densas e subcapsular posterior e opacidades vítreas ${ }^{(20-24)}$.

Outras alterações oculares, ocasionadas por modificações degenerativas e patológicas ou por intervenções terapêuticas, não alteram significativamente seus resultados. Exemplos são cataratas pouco densas, cirurgias refrativas (PRK ou LASIK), implantes de lentes intra-oculares, vitrectomias com óleo de silicone e o uso de lentes de contato gelatinosa ou rígida $^{(2,25-28)}$.

Como a catarata e o glaucoma são comuns e freqüentemente estão associadas na senilidade, muitas vezes é necessária a realização do GDx nesses pacientes. No seguimento dos mesmos, a cirurgia de catarata é frequentemente indicada.

A opacificação de cápsula posterior (OCP), complicação mais freqüente da cirurgia de catarata, torna-se, portanto, uma ocorrência comum em pacientes glaucomatosos. Pelo risco do GDx, arma importante no diagnóstico precoce dos pacientes com suspeita de glaucoma, obter uma informação inadequada em pacientes pseudofácicos com OCP, foi gerada a necessidade de realizar este estudo.

Nos olhos em que a OCP é muito intensa (Grau 5 e alguns Grau 4), o GDx não conseguiu formar imagem satisfatória. No entanto, em todos aqueles em que se obteve imagem analisável dentro dos parâmetros de aquisição do aparelho, a presença da $\mathrm{OCP}$, que embora reduza de forma significante a acuidade visual, não influenciou no resultado de nenhuma de suas variáveis, não havendo diferença significante antes e após a realização da capsulotomia com Nd: YAG laser (Tabela 1).

\section{CONCLUSÃO}

A presença de OCP em graus leves/moderados em pacientes pseudofácicos não influencia nos resultados da análise das imagens das fibras do nervo óptico pelo GDx. Apenas intensos graus de OCP que impeçam a obtenção de imagem analisável pelo aparelho inviabilizam o exame.

\section{ABSTRACT}

Purpose: To evaluate if posterior capsule opacification (PCO) would alter the analysis of nerve fiber layer using the scanning laser polarimetry (GDx). Methods: Pseudophakic non- 
glaucomatosous eyes (37 patients) underwent nerve fiber layer analysis using GDx before and after posterior capsulotomy with Nd:YAG Laser. Results: In five eyes, GDx did not obtain acceptable images using the device's parameters of acquisition. In the others, mean variable values did not reach significant difference before and after posterior capsulotomy. Conclusion: Moderate degrees of PCO and/or acceptable images in pseudophakic patients do not alter the analysis of nerve fiber layer by GDx. Only intense degrees of PCO that hinder analyzable images make the examination impracticable.

Keywords: Pseudophakia; Glaucoma; Nerve fibers/pathology; Diagnostic techniques, ophthalmological; Lasers/diagnostic use

\section{REFERÊNCIAS}

1. Almeida HG. Cirurgia combinada: facoemulsificação e trabeculectomia. In: Resende F, organizador. Cirurgia da catarata. $2^{\underline{a}}$ ed. Rio de Janeiro: Cultura Médica; 2002. p.373-9

2. Kremmer S, Pflug A, Heiligenhaus A, Fanihagh F, Steuhl KP. ScanningLaser-Topometrie und Polarimetrie vor und nach Katarakt-Operationen mit Implantation von Intraokularlinsen. Klin Monatsbl Augenheilkd. 1999; 214(6):378-85.

3. Spalton DJ. Posterior capsular opacification after cataract surgery. Eye. 1999; 13(Pt 3b):489-92. Review.

4. Ram J, Kaushik S, Brar GS, Gupta A. Neodymium: YAG capsulotomy rates following phacoemulsification with implantation of PMMA, silicone, and acrylic intraocular lenses. Ophthalmic Surg Lasers. 2001;32(5):375-82.

5. Marcantonio JM, Vrensen GF. Cell biology of posterior capsular opacification. Eye. 1999;13(Pt 3b):484-8.

6. Emery J. Capsular opacification after cataract surgery. Curr Opin Ophthalmol. 1999;10(1):73-80. Review.

7. Nishi O. Posterior capsule opacification. Part 1: Experimental investigations. J Cataract Refract Surg. 1999;25(1):106-17. Review.

8. Schaumberg DA, Dana MR, Christen WG, Glynn RJ. A systematic overview of the incidence of posterior capsule opacification. Ophthalmology. 1998; 105(7):1213-21.

9. Airaksinen PJ, Alanko HI. Effect of retinal nerve fibre loss on the optic nerve head configuration in early glaucoma. Graefes Arch Clin Exp Ophthalmol. 1983;220(4):193-6.

10. Tuulonen A, Lehtola J, Airaksinen PJ. Nerve fiber layer defects with normal visual fields. Do normal optic disc and normal visual field indicate absence of glaucomatous abnormality? Ophthalmology. 1993;100(5):587-97; discussion 597-8.
11. Colen TP, Tjon-Fo-sang MJ, Mulder PG, Lemij HG. Reproducibility of measurements with the nerve fiber analyzer (NfA/GDx). J Glaucoma. 2000; 9(5):363-70.

12. Fabré K, Michiels I, Zeyen T. The sensitivity and specificity of TOP, FDP and GDX in screening for early glaucoma. Bull Soc Belge Ophtalmol. 2000; 275:17-23.

13. Weinreb RN, Zangwill L, Berry CC, Bathija R, Sample PA. Detection of glaucoma with scanning laser polarimetry. Arch Ophthalmol. 2000;116(12): 1583-9.

14. Sinai MJ, Essock EA, Fechtner RD, Srinivasan N. Diffuse and localized nerve fiber layer loss measured with a scanning laser polarimeter: sensitivity and specificity of detecting glaucoma. J Glaucoma. 2000;9(2):154-62.

15. Choplin NT, Lundy DC. The sensitivity and specificity of scanning laser polarimetry in detection of glaucoma in a clinical setting. Ophthalmology. 2001;108(5):899-904. Comment in: Ophthalmology. 2002;109(2):216-7. Ophthalmology. 2003;110(9):1858; author reply 1858.

16. Yamada N, Chen PP, Mills RP, Leen MM, Stamper RL, Lieberman MF, et al. Glaucoma screening using the scanning laser polarimeter. J Glaucoma. 2000;9(3):254-61.

17. Hoh ST, Greenfield DS, Liebmann JM, Maw R, Ishikawa H, Chew SJ, Ritch R. Factors affecting image acquisition during scanning laser polarimetry. Ophthalmic Surg Lasers. 1998;29(7):545-51.

18. Giampani Júnior J, Leal BC, Susanna Júnior R. Analisador de fibras nervosas: um estudo sobre os resultados falso-positivo. Arq Bras Oftalmol. 2001; 64(1):9-12.

19. Collur S, Carroll AM, Cameron BD. Human lens effect on in vivo scanning laser polarimetric measurements of retinal nerve fiber layer thickness. Ophthalmic Surg Lasers. 2000;31(2):126-30.

20. Holló G. Factors affecting image acquisition during scanning laser polarimetry. Ophthalmic Surg Lasers. 1999;30(1):74.

21. Park RJ, Chen PP, Karyampudi P, Mills RP, Harrison DA, Kim J. Effects of cataract extraction with intraocular lens placement on scanning laser polarimetry of the peripapillary nerve fiber layer. Am J Ophthamol. 2001;132(4): 507-11.

22. Lima MS, Zangwill L, Weinred RN. Scanning laser polarimetry to assess the nerve fiber layer. In: Schuman JS, editor. Imaging in glaucoma. Thorofare, NJ: Slack; c1997. p. 83-92.

23. Pons ME, Rothman RF, Ozden RG, Liebmann JM, Ritch R. Vitreous opacities affect scanning laser polarimetry measurements. Am J Ophthalmol. 2001;131(4):511-3.

24. Optic nerve head and retinal nerve fiber layer analysis. American Academy of Ophthalmology. Ophthalmology. 1999;106(7):1414-24.

25. Choplin NT, Schallhorn SC. The effect of excimer laser photorefractive keratectomy for myopia on nerve fiber layer thickness measurements as determined by scanning laser polarimetry. Ophthalmology. 1999;106(5): 1019-23. Comment in: Ophthalmology. 2000;107(6):1021-2.

26. Bhandari A, Chen PP, Mills RP. Effects of contact lenses on scanning laser polarimetry of the peripapillary retinal nerve fiber layer. Am J Ophthalmol. 1999;127(6):722-4.

27. Gürses-Ozden R, Pons ME, Barbieri C, Ishikawa H, Buxton DF, Liebmann JM, Ritch R. Scanning laser polarimetry measurements after laser-assisted in situ keratomileusis. Am J Ophthalmol. 2002;129(4):461-4.

28. Holló G, Bereczki A, Milibák T, Süveges I. Scanning laser polarimetry via intravitreal silicone oil. Acta Ophthalmol Scand. 1999;77(5): 519-21.

\section{Agradecimento}

Agradeço ao colega Dario Augusto Bender Moreira (Ariquemes, Rondônia) por seu gentilíssimo questionamento sobre a dosagem de levodopa no tratamento da ambliopia (como eventual adjuvante), cuja indicação, feita no Programa de Educação Continuada (PEC) do C.B.O., em seu CD número 9, contém um erro, lamentavelmente inadvertido na edição da gravação.

De fato, ao invés de se recomendar a posologia de $0,70 \mathrm{mg} / \mathrm{kg}$ peso/dia ${ }^{(1)}$ e embora dosagens bem maiores do que esta já tenham sido empregadas no tratamento da ambliopia ${ }^{(2)}$, indicou-se, por um lapso, uma cem vezes maior (70mg/kg peso/dia). Comercialmente, a levodopa pode ser encontrada em drágeas ou comprimidos nas apresentações de 100, 250 e 500 mg. Para outras condições (discinesias, parkinsonismo) referem-se dosagens iniciais de 500 a $1000 \mathrm{mg} / \mathrm{dia}$, divididas em duas ou mais vezes, admitindo-se que a administração terapêutica ótima e costumeira possa aumentar, ainda que não deva exceder $8000 \mathrm{mg} / \mathrm{dia}^{(3)}$.

\section{REFERÊNCIAS}

1. Procianoy E, Procianoy L, Procianoy F. Resultados do tratamento da ambliopia com levodopa combinada à oclusão. Arq Bras Oftalmol. 2004;67(5):717-20.

\begin{abstract}
2. Procianoy E, Fuchs FD, Procianoy F, Procianoy L. Uso de levodopa em pacientes com ambliopia. Arq Bras Oftalmol. 2000;63(5):399-402.

3. Fraunfelder FT, Roy FH. Current Ocular Therapy. U.S.A.: WB. Saunders; 1995. p.876.
\end{abstract}

Harley E. A. Bicas 